\title{
Coordination entre production et réception :
}

de l'interaction directe à la quasi-interaction médiée

\section{Philippe Ross}

\section{(2) OpenEdition \\ Journals}

Édition électronique

URL : http://journals.openedition.org/ticetsociete/1282

DOI : 10.4000/ticetsociete. 1282

\section{Éditeur}

Association ARTIC

\section{Référence électronique}

Philippe Ross, «Coordination entre production et réception : », tic\&société [En ligne], Vol. 7, № 1 | 1er semestre 2013, mis en ligne le 04 juin 2013, consulté le 22 avril 2019. URL : http:// journals.openedition.org/ticetsociete/1282 ; DOI : 10.4000/ticetsociete.1282 


\section{Coordination entre production et réception :}

de l'interaction directe à la quasi-interaction médiée

Philippe Ross, docteur en Médias et communication (London School of Economics), est Professeur adjoint au Département de communication de l'Université d'Ottawa depuis 2008. II travaille au carrefour des théories des médias et de la sociologie des sciences et techniques. II s'intéresse particulièrement au rôle de la connaissance et de l'expérience sociale dans le façonnement des relations producteurs-publics. Ses recherches, qui s'attaquent à ces questions tant dans leurs manifestations empiriques que leurs implications théoriques, ont été publiées dans des revues telles que Social Studies of Science, New Media \& Society, Television and New Media, et Sociology Compass. 
Philippe ROSS

\section{Résumé}

Les nouvelles formes de collaboration qui accompagnent le Web 2.0 favorisent la consolidation d'une fâcheuse tendance en recherches sur les médias et la technologie: celle qui consiste à réifier le public et à en surestimer l'implication directe en production. Bien que les exemples de collaboration effective entre producteurs et récepteurs/usagers se multiplient, ils ne constituent pas pour autant la règle. Dès lors, la nature de la production en tant qu'activité sociale foncièrement orientée vers le public doit-elle conduire à favoriser le rôle actif de ce dernier en production? Cet article propose une réflexion théorique sur la coordination entre les sphères de la production et de la réception/de l'usage qui résiste à la réification du public et au modèle d'interaction directe entre producteurs et publics qui l'accompagne. II offre, dans un premier temps, un survol critique de quelques approches influentes de la production technologique et médiatique qui font une grande place au rôle actif des publics et à la connaissance que les producteurs dérivent de leurs interactions directe avec ces derniers. L'article développe ensuite une conception du rapport producteurs-publics en tant que "quasi-interaction médiée » (Thompson, 1995), afin de refléter plus fidèlement le processus par lequel les producteurs représentent le public en son absence, leur recours à l'expérience sociale ordinaire ce faisant, et la nature de l'auditoire en tant que cause et conséquence de la production.

Mots-clés : production, réception, interaction producteurs/usagers, public imaginé, médiation

\section{Abstract}

The new forms of collaboration that are a feature of Web 2.0 exacerbate the unfortunate tendency, in research on media and technology, to reify the public and to overstate its direct involvement in production. Although effective collaboration between producers and audiences/user is increasingly common, it is not yet the norm. It is worth asking, therefore, whether a conception of production as a social activity oriented to an audience must entail an emphasis on its active role. This theoretical essay addresses the coordination of the spheres of production and reception/usage in a way that resists both the reification of the public and the model of direct interaction that follows from it. This paper offers, first, a critical overview of influential approaches to technological and media production which focus on the public's active role and on the knowledge producers derive from their direct interactions with its members. It then goes on to develop a conception of the relationship between producers and their publics as a form of 'mediated 
Coordination entre production et réception :

de l'interaction directe à la quasi-interaction médiée

quasi-interaction' (Thompson, 1995), as a means of better reflecting the process by which producers represent the public in its absence, their recourse to ordinary social experience in so doing, and the nature of the audience as both the cause and consequence of production.

Keywords: media production, reception, producer-user interaction, imagined public, mediation

\section{Resumen}

Las nuevas formas de colaboración que acompañan la Web 2.0 favorecen la consolidación de una tendencia problemática en las investigaciones sobre los medios y las tecnologías: la que consiste en reificar el público, sobreestimando así su implicación directa en la producción. Si bien los ejemplos de colaboración efectiva entre productores y receptores/usuarios se multiplican, éstos constituyen más la excepción que la regla. Cabe preguntarse entonces si la naturaleza de la producción, como actividad social orientada primeramente hacia el público, debe favorecer el papel activo de éste en la producción. Este artículo propone una reflexión teórica sobre la coordinación entre las esferas de la producción y de la recepción/uso que resiste justamente a esta idealización del público y al modelo de interacción directa entre productores y públicos, que le acompaña. En un primer tiempo, se presenta una revisión crítica de determinados enfoques que influyen en la producción tecnológica y mediática, y que asignan un papel activo a los públicos y al conocimiento que los productores derivan de sus interacciones directas con ellos. Posteriormente, el artículo desarrolla una concepción de la relación productores-públicos, en tanto que "cuasi-interacción mediada" (Thompson, 1995), para reflejar, de forma fiel, el proceso a través del cual los productores representan al público, recurriendo a la experiencia social ordinaria, así como la naturaleza de la audiencia, como causa y consecuencia de la producción.

Palabras clave: producción, recepción, interacción productores-usuarios, público imaginado, mediación 
Philippe ROSS

\section{Introduction}

La vigueur avec laquelle le public a investi l'espace médiatique par le biais de blogues, sites de réseautage social et autres rubriques «à vous la parole » a de quoi convaincre même le relativiste le plus endurci que ces technologies numériques répondent à un besoin que des inventeurs de génie ont eu la clairvoyance de reconnaitre. Comment expliquer un tel engouement pour les nouvelles plateformes de communication de masse sinon par un désir profond d'expression, d'interaction et d'échange entre semblables plus ou moins dispersés dans le temps et l'espace ? En tout cas, l'alternative qui consisterait à invoquer l'enrôlement du public (Callon, 1986; Ross, 2011a) dans une entreprise dont les bénéfices pour lui ne seraient pas donnés mais plutôt construits socialement, ne semble guère plus satisfaisante à la lumière de la vitesse de diffusion fulgurante des médias sociaux. Le public ne s'est pas fait prier pour les adopter; pas plus que les plateformes telles Facebook, Google, YouTube et Twitter n'ont encore déterminé comment - voire si - leur potentiel commercial peut se réaliser.

Quoi qu'il en soit, le paysage médiatique qui se dessine donne l'impression d'une coordination accrue entre l'offre et la demande, la production et la réception, les producteurs et leurs publics. En effet, si la sphère de production médiatique a toujours eu une relation d'influence réciproque avec celle de la réception qu'elle cherche à la fois à comprendre et à informer, cette relation s'opère désormais en temps réel ou presque grâce aux plateformes, services et algorithmes par lesquels on s'assure le concours - plus ou moins délibéré ou informé - d'individus si prompts à exprimer leurs goûts, intérêts et états d'âme. Ceci suscite tant l'inquiétude chez ceux qui y voient l'initiative d'industriels cherchant à consolider leur emprise sur la production par la marchandisation des récepteurs de leurs créations (Pariser, 2011; Turow, 2012), qu'une célébration de la créativité et l'autonomie de nouveaux acteurs (Jenkins, 2006) à même de déstabiliser l'ordre établi en matière de culture, de politique et de commerce. Quel que soit le point de vue, les nouveaux dispositifs du Web 2.0 sont portés par une logique simple : les membres du public ont quelque chose de valeur à communiquer à autrui (membres du public, producteurs ou publicitaires) que les producteurs, seuls, ne peuvent offrir (Ross, 2012b). A priori raisonnable, cette logique favorise pourtant la consolidation d'une fâcheuse tendance en recherches sur la production : celle qui consiste à réifier le public et à en surestimer l'implication directe en production.

II n'est pas surprenant que les praticiens envisagent leurs publics comme des groupes empiriquement réels dont l'existence se vérifie à coups de sondages, études de marché, focus groups et cotes d'écoute. Ou qu'ils 
Coordination entre production et réception :

de l'interaction directe à la quasi-interaction médiée

cherchent à les connaître toujours mieux grâce aux nouveaux mécanismes de rétroaction et de forage de données (Bennett et Manheim, 2006), sous le prétexte d'engager une grande conversation à même de corriger l'asymétrie qui caractérise traditionnellement leur relation. Nombre de théoriciens des médias (Thompson, 1995; McQuail, 1987) et de chercheurs dans le champ émergent des "production studies " (Caldwell, 2008; Hesmondhalgh et Baker, 2011; Mayer, 2011) ont souligné l'incertitude profonde qui caractérise le travail des acteurs de la production eu égard au public, ainsi que les moyens qu'ils mobilisent pour établir un sentiment de proximité avec ce dernier (Ross, 2012a; Hermida et Thurman, 2008).

D'autres ont montré que, en dépit des efforts pour engager le public au moment de la production, celui-ci demeure difficile à cerner (Jouët, 2003; Hine, 2001), voire une construction sociale - c'est-à-dire une réalité empirique vérifiable seulement a posteriori, en l'occurrence au moment de la consommation (Gitlin, 1983). C'est le cas, notamment, de recherches en communication (e.g. Ross, 2011a) inspirées des travaux en sociologie des sciences et techniques sur la représentation des usagers en production (Akrich, 1992; 1995; Bardini, 2000; Pinch et Oudshoorn, 2003). Or, cette perspective qui aborde le public comme objet plutôt que comme sujet de la coordination en production demeure sous-développée - et le contexte médiatique actuel n'est pas propice à un renversement de situation. Au contraire, avec le crowdfunding, le crowdsourcing ou la " collaboration de masse " (Tapscott et Williams, 2010), le Web 2.0 promet aux usagers un rôle plus important que jamais dans ce qu'on décrit comme «a new approach to orchestrating capability to innovate, create goods and services, and solve problems. Social networking is becoming social production, where self-organizing groups of peers can design and produce everything from software to motorcycles » (Tapscott et Williams, 2010: pp. 2627).

Les exemples de telles collaborations se multiplient mais elles ne constituent pas pour autant la règle - pas plus pour la production de technologies ou de médias que pour celle de motocyclettes. Dès lors, il y a lieu de poser la question : la nature de la production en tant qu'activité sociale foncièrement orientée vers le public doit-elle conduire à favoriser le rôle actif de ce dernier en production?

Cet article propose une réflexion théorique sur la coordination entre les sphères de la production et de la réception/de l'usage qui résiste à la réification du public et au modèle d'interaction directe entre producteurs et publics qui l'accompagne. II offre, dans un premier temps, un survol critique de quelques approches influentes de la production technologique et médiatique, qui font une 
Philippe ROSS

grande place au rôle actif des publics et à la connaissance que les producteurs dérivent de leurs interactions directe avec ces derniers. L'article développe ensuite une conception du rapport producteurs-publics en tant que «quasiinteraction médiée » (Thompson, 1995), afin de refléter plus fidèlement le processus par lequel les producteurs représentent le public en son absence, leur recours à l'expérience sociale ordinaire ce faisant, et la nature de l'auditoire en tant que cause et conséquence de la production.

\section{Le public actif et le biais de l'interaction directe}

En sociologie des sciences et techniques, on reconnaît depuis longtemps le rôle déterminant des usagers dans le sort des artéfacts qui atteignent le marché. Or, plus récemment - mais avant l'arrivée du Web 2.0 ! - l'influence des usagers s'est propagée en amont dans le «cycle de vie » de la technologie (Flichy, 1995) pour atteindre la production. Nombre d'approches dites participatives envisagent désormais les usagers comme acteurs à part entière de l'innovation (Akrich, 1998), si bien que les études qui s'intéressent à la mise en forme d'artéfacts nouveaux mettent bien souvent ces acteurs à l'avant-plan, au détriment des producteurs.

C'est le cas de recherches qui portent ostensiblement sur la conception ou la production d'artéfacts mais qui, en pratique, concernent leur mise en œuvre - la zone grise dans le cycle de vie des innovations dans laquelle conception et usage se chevauchent et où l'usager joue plus ou moins explicitement le rôle de concepteur. On s'intéresse ainsi au « design-in-use » (Lievrouw, 2002 : p.184), signifiant par là que la mise en forme matérielle des artéfacts continue une fois que ceux-ci sont entre les mains des usagers (Brown and Duguid, 1994; Star and Bowker, 2002). Certains, comme Star et Bowker (2002, p.159), vont même jusqu'à affirmer que " the work of design is in many ways secondary to the work of modification ", accordant ainsi aux usagers le dernier mot dans la mise en forme technique. Ce serait en effet l'usager qui, ici, assurerait la coordination entre les sphères de production et de réception, en validant ou infirmant les décisions prises en amont par les producteurs et cristallisées en un prototype ou artéfact plus ou moins abouti.

D'autres vont encore plus loin dans cette direction. Par exemple, tant les études en Human-Computer Interaction et en Computer Supported Cooperative Work (e.g. Beirne et al., 1998; Mackay et al., 2000), que celles s'intéressant à l'innovation organisationnelle (e.g. Fulk et DeSanctis, 1995; Monge et al., 1998), supposent un chevauchement effectif entre concepteurs et usagers. En effet, elles concernent l'innovation à l'intention de groupes circonscrits (organisations ou communautés établies) et dont les membres sont - logiquement - enrôlés comme concepteurs dans le processus. Ces études sont donc, en fait, des cas 
Coordination entre production et réception :

de l'interaction directe à la quasi-interaction médiée

de technologies-sur-mesure dont les leçons peuvent difficilement s'appliquer au développement d'artéfacts pour un public de masse, c'est-à-dire un groupe qui ne peut être observé ou engagé comme tel.

En somme, l'interaction directe avec les usagers est certes valable en tant que méthodologie du design ou comme principe normatif. Mais la perspective de "l'usager-concepteur » (" users-as-designers »; Ross, 2011a) comporte au moins deux faiblesses importantes eu égard à leur capacité d'analyse critique de l'interface entre production et réception. Premièrement, elle peut donner l'impression d'une distribution équitable de pouvoir entre concepteurs et usagers. Or, même si la production est une entreprise orientée vers un marché (Lundvall, 1992) ou un public anticipé (Ross, 2012a) et que les usagers sont sollicités au cours du processus d'innovation, ils en sont rarement des agents pleinement informés - même dans le contexte de Web 2.0 (Pariser, 2011; Turow, 2012). Deuxièmement (et plus important encore à notre propos), parce qu'elle favorise le rôle d'usagers "réels" (plutôt que "sémiotiques "; Oudshoorn et al., 2004) dans la mise en forme technique, ${ }^{1}$ la perspective de "l'usager-producteur " donne à croire que l'environnement contrôlé du laboratoire ou des essais sur le terrain est le lieu privilégié sinon unique de la rencontre entre les sphères de la production et de l'usage. En effet, elle suppose que les situations sociales les plus aptes à générer des connaissances sur l'usager sont celles - artificielles - conçues par les producteurs pour permettre l'interaction directe avec les usagers dans le cadre d'un projet technique spécifique. Elle fait peu de place aux interactions qui s'exerceraient à l'extérieur de ce cadre et dont l'influence peut se manifester en production.

Cette dernière critique s'applique également à la conception du rapport producteur-public en études des médias, qui tendent elles aussi à idéaliser le rapport direct entre producteurs et membres du public « réels» ou « effectifs ». C'est le cas des «production studies" (Caldwell, 2008; Mayer et al., 2009; Mayer, 2011; Hesmondhalgh et Baker, 2011), ce champ en pleine émergence qui cherche à revaloriser l'étude de la production en privilégiant l'ethnographie et "l'expérience vécue " (lived experience) des artisans, professionnels et cadres qui y oeuvrent. Ici, on fait grand cas du peu d'interaction directe et continue entre les producteurs et leurs publics. Par exemple, Zafirau (2009, p.194) décrit le "sentiment de distance sociale ", en termes de classe et de géographie, que ressentent les professionnels de l'industrie du cinéma par rapport à leur public. Hesmondhalgh et Baker (2011) relatent quant à eux un problème unique aux industries culturelles : alors que celles ci-reposent, plus

\footnotetext{
${ }^{1}$ Par exemple, des « usagers de pointe » (von Hippel, 1986) ou des « usagers ambassadeurs » (Mackay et al., 2000).
} 
Philippe ROSS

que toute autre organisation, sur des actes de communication, «this often consists of communication with groups of people with whom the creative worker is likely to have little interaction » (Hesmondhalgh et Baker 2011, p.200). Ils ajoutent: «[c]reative workers are rarely co-present with their audiences, except in live performances» (ibid.). Ce manque d'interaction est une source “ d'anxiété, d'ambivalence et de méfiance » (Hesmondhalgh et Baker, 2011, p.200) parmi les producteurs professionnels; elle incarne le risque inhérent à la production culturelle de masse (Deuze, 2007, p.65); elle est au cœur des luttes de pouvoir en production (Caldwell, 2008, p.199) et elle fait de la production une activité foncièrement « spéculative » (Caldwell, 2008, p.22).

Une telle idéalisation de l'interaction directe n'est pas exceptionnelle. Elle fait écho aux théoriciens de la «médiation » (Ross, 2012b; Couldry, 2008; Silverstone, 2005; Kauffman, 2008) - le rôle de charnière joué par les médias entre l'expérience individuelle et la réalité collective - et particulièrement l'œuvre influente du sociologue John B. Thompson. Pour Thompson (1995), la communication de masse se caractérise par la cassure structurée (structured break) entre la production et la réception de biens symboliques et qui fait en sorte que les producteurs « are generally deprived of the direct and continuous forms of feedback characteristic of face-to-face interaction. Hence the processes of production and transmission are characterized by a distinctive kind of indeterminacy, since these processes take place in the absence of cues provided by recipients. » (Thompson, 1995, p.29)

Le manque de co-présence entre producteurs et récepteurs effectifs équivaut donc, dans ces approches de la production médiatique, à une absence de relation. Les producteurs seraient, par défaut, dans un état de parfaite incertitude (ou ignorance !) par rapport aux récepteurs éventuels de leurs créations puisque privés d'indices quant aux préférences et comportements de ces derniers. Cet état présumé exclut de facto la possibilité que les producteurs sachent des choses au sujet des récepteurs qu'ils auraient acquises dans des situations «naturelles ». II conduit ainsi à accorder une importance démesurée à toute information obtenue auprès de récepteurs en chair et en os par le bais de sondages, focus groups ou autres mesures d'audience, comme si celles-ci avaient une valeur absolue - comme si elles constituaient des connaissances au sujet $d u$ public. Or, exprimer un souci pour le public et ses besoins est une chose; les traiter comme une réalité saisissable en est une autre.

Selon Stewart et Williams (2005), les praticiens du design centré sur l'usager (user-centred design) perpétuent la présomption fallacieuse que le succès de tout projet tient à l'accumulation de « ever more extensive knowledge about the specific context and purposes of various users into technology design » (Stewart et Williams, 2005, p.4). Selon nous, les chercheurs qui privilégient l'interaction 
Coordination entre production et réception :

de l'interaction directe à la quasi-interaction médiée

directe entre producteurs et usagers/récepteurs commettent la même faute que les praticiens qu'ils observent - ils deviennent eux-mêmes des «indigènes »! En effet, en favorisant l'étude de l'appropriation d'artéfacts et de contenus, les approches discutées précédemment mobilisent une conception strictement instrumentale du rapport qu'ont les producteurs aux usagers, et de la connaissance qui est valorisée en production. Elles dépeignent la production comme une entreprise capable de solliciter des usagers/récepteurs présumés réels et dont les besoins le sont tout autant; et les producteurs comme des individus dont le rapport au monde social (y compris les espaces de la réception) se limite au contexte institutionnel à l'étude. Dans la mesure où elles prétendent s'intéresser aux dynamiques sociales de la production de biens matériels ou symboliques, ces études affichent une surprenante réticence à "suivre les acteurs " (Latour, 1989) qui invitent, simulent, et dans tous les cas encadrent, toute contribution du public en production - c'est à dire les producteurs.

Les ethnographes de la production pourraient bien invoquer des contraintes méthodologiques pour justifier un tel biais : comment vérifier l'existence d'une relation entre producteur et récepteur/ usager sinon en observant leurs interactions effectives ? Mais une telle explication ne saurait tenir la route, surtout avec l'émergence de l'ethnographie «multi-site", dont l'opérationnalisation convient à la production :

'Within a single site, the crucial issue concerns the detectable system-awareness in the everyday consciousness and actions of subjects' lives. This is not an abstract theoretical awareness such as social scientists might seek, but a sensed, partially articulated awareness of specific other sites and agents to which particular subjects have (not always tangible) relationships' (Marcus, 1998, p.96).

Ainsi, la reconnaissance de l'importance des usagers ne doit pas obligatoirement se traduire en un biais méthodologique favorable aux usagers "réels" et aux contextes d'usage effectif. Dans les études discutées précédemment, le statut des usagers comme ultimes arbitres du sort d'artéfacts nouveaux incite les chercheurs à traiter de leur rôle (actif) dans la "stabilisation matérielle» («material closure»; Pinch et Bijker, 1984) de technologies. Pourtant, les débats et discussions qui ont lieu en production invitent à analyser leur rôle (passif) dans la " stabilisation rhétorique » (« rhetorical closure »; ibid.), c'est-à-dire la façon dont le public invoqué - qui peut découler d'une prise en compte sincère de besoins et/ou d'attentes mais qui n'est jamais détaché de la perception du producteur - permet aux producteurs de dissiper l'incertitude et de prendre des décisions en production. 


\section{Représentation des récepteurs/usagers et quasi- interaction médiée}

L'appropriation d'artéfacts et de contenus n'est qu'une des arènes dans lesquelles se déroule «l'apprentissage social » nécessaire à l'innovation (Williams et al., 2005, p.58).2 Selon Lundvall, les systèmes d'innovation sont des systèmes sociaux et dynamiques, marqués par la rétroaction et par la "reproduction of the knowledge of individuals or collective agents (through remembering) » (Lundvall, 1992, p.2). Dans ce contexte, "[a]lmost all innovations reflect already existing knowledge, combined in new ways " (ibid. p.8) et la coordination entre production et usage est conçue comme relevant d'un processus «d'apprentissage interactif ou social " (interactive or social learning) (Edquist, 1997; Lundvall, 1992; Williams et al., 2005). Le concept d'apprentissage interactif ou social repose sur deux propositions : d'abord, « (...) the most fundamental resource in the modern economy is knowledge and, accordingly, the most important process is learning "; ensuite, "learning is predominantly an interactive and, therefore, a socially embedded process which cannot be understood without taking into consideration its institutional and cultural context » (Lundvall, 1992, p.1). Une telle conception rejette la raison instrumentale associée aux acteurs de la production et attire l'attention sur l'importance du contexte culturel, notamment sous la forme de l'expérience sociale ordinaire des acteurs impliqués dans le processus d'innovation.

Outre l'arène de l'appropriation, l'apprentissage social se manifeste dans la représentation des usagers à l'étape de la conception (Williams et al. 2005, p.58). Celle-ci nous semble un garde-fou essentiel contre la réification du public et ses écueils car elle permet une conceptualisation plus inclusive des connaissances mobilisées en production pour assurer sa coordination avec la sphère de la réception/ de l'usage, sans pour autant exclure de penser ce rapport en termes d'interaction sociale. La discussion qui suit en fait la démonstration en trois temps. D'abord, elle avance une conception du rapport producteurs-publics en tant que "quasi-interaction médiée », que Thompson (1995) décrit comme un type d'interaction propre à la communication de masse. À la différence de Thompson, toutefois, nous employons le concept pour penser

2 Ce concept fécond vient de l'approche des Systèmes d'innovation (Systems of Innovation), qui prend pour objet les canaux de communication liant la production et la consommation de produits nouveaux. Comme l'explique Lundvall (1992), cette approche marque une rupture avec « la raison instrumentale et stratégique » (p.47) qui caractérise la coordination entre production et usage dans les modèles néo-classiques de l'innovation, en particulier la conception de l'entrepreneur de Schumpeter (1934). Les études sur l'appropriation d'artéfacts et de contenus, comme celles discutées dans la section précédente, illustrent «l'apprentissage par l'usage » (learning-by-using) (Williams et al., 2005, p.58). 
Coordination entre production et réception :

de l'interaction directe à la quasi-interaction médiée

la communication de masse telle qu'elle est opérée par les producteurs, plutôt que par les récepteurs ou usagers. Ceci est en phase avec notre posture épistémologique constructiviste, selon laquelle les producteurs d'un artéfact en développement en sont les seuls agents "délibérés » ou pleinement informés -les récepteurs ou usagers ne pouvant intervenir, de manière active, qu'une fois le dispositif suffisamment stabilisé. La discussion met ainsi l'accent, dans un second temps, sur la réflexivité, la familiarité et les connaissances tacites des producteurs, qui sont autant d'indicateurs d'une relation avec le public établie bien en amont du contexte de production. Elle souligne enfin la nature circulaire de cette relation, et ses conséquences eu égard à la théorisation de la production.

\subsection{La quasi-interaction médiée}

Dans The Media and Modernity, Thompson (1995) présente une typologie des formes d'interaction sociale qui comprend l'interaction directe, l'interaction médiée et la quasi-interaction médiée. II affirme que cette dernière forme d'interaction a accompagné le développement des médias de masse et que, par conséquent, elle est propre à la modernité. II écrit :

The development of new media of communication does not consist simply in the establishment of new networks for the transmission of information between individuals whose basic social relationships remain intact. Rather, the development of communication media creates new forms of action and interaction and new kinds of social relationships - forms which are quite different from the kind of faceto-face interaction which has prevailed for most of human history. It also brings about a complex reordering of patterns of human interaction across space and time. With the development of communication media, social interaction is separated from physical locale, so that individuals can interact with one another even though they do not share a common spatio-temporal setting. The use of communication media gives rise to new forms of interaction which are extended in space (and perhaps also in time), and which display a range of characteristics that differentiate them from faceto-face interaction. (Thompson, 1995, p.82)

Bien que Thompson cherche à expliquer comment l'usage des médias de communication affecte les relations sociales, son analyse s'intéresse peu aux affordances de technologies spécifiques. Elle s'attarde plutôt à la façon dont les formes existantes d'action et d'interaction s'accommodent des formes émergentes. Ainsi, la quasi-interaction médiée se distinguerait des deux formes 
précédentes - l'interaction en face-à-face et l'interaction médiée - suivant deux aspects :

In the first place, the participants in face-to-face interaction and mediated interaction are oriented towards specific others, for whom they produce actions, utterances, etc.; but in the case of mediated quasi-interaction, symbolic forms are produced for an indefinite range of potential recipients. Second, whereas face-toface interaction and mediated interaction are dialogical, mediated quasi-interaction is monological in character, in the sense that the flow of communication is predominantly one-way. The reader of a book, for instance, is primarily the recipient of a symbolic form whose producer does not require (and generally does not receive) a direct and immediate response. (Thompson, 1995, p.84)

La rapport entre communicateurs de masse et leurs publics serait une quasiinteraction - et non pas une interaction proprement dite - en raison de son caractère monologique, et parce qu'il concerne la production de contenus symboliques à l'intention d'un groupe de récepteurs aux contours indéfinis. Cette quasi-interaction crée néanmoins " a certain kind of social situation in which individuals are linked together in a process of communication and symbolic exchange » (Thompson 1995, p.84). Les situations sociales ainsi désignées sont des situations structurées dans lesquelles « some individuals are engaged primarily in producing symbolic forms for others who are not physically present, while others are involved primarily in receiving symbolic forms produced by others (...) 》 (Thompson, 1995, p.84).

II serait tentant d'invoquer la «culture participative » (Jenkins, 2006) et le « public créatif » (Castells 2009) qui accompagnent le Web 2.0 pour contester la relation monologique décrite par Thompson, ainsi que le caractère flou du public et la nette distinction qu'il fait entre contextes de la production et ceux de la réception. Or, plusieurs recherches récentes sur les blogueurs et usagers de sites de réseautage social incitent à la prudence à cet égard. Par exemple, Brake (2009 , p.3) a trouvé que les blogueurs « have limited interest in gathering information about their readers » et qu'ils se fient à leur imagination et à des présomptions quant à leurs publics afin de donner sens à leurs pratiques communicationnelles. Dans la même veine, Marwick et boyd (2011) soutiennent qu'il est impossible pour les usagers de Twitter « to vary self-presentation based on audience » parce que la diversité potentielle des publics sur Twitter en fait, en pratique, un groupe inconnu (Marwick et boyd, 2011, p.3). En somme, la connaissance de son public apparaît comme inatteignable ou simplement superflue et le mieux que les usagers de tels médias puissent espérer, c'est de glaner des indices de l'environnement en-ligne afin de s'imaginer la 
Coordination entre production et réception :

de l'interaction directe à la quasi-interaction médiée

communauté (boyd 2007, p.131). Ainsi, de telles études postulent aussi que les communicateurs de masse sont dans un état d'ignorance par défaut, lequel ne peut être surmonté que par le biais d'une interaction directe avec les membres du public effectifs.

Suivant Thompson, la quasi-interaction médiée est une forme d'« action à distance " qui permet aux individus " to act for others who are dispersed in space and time, as well as enabling individuals to act in response to actions and events taking place in distant locales " (Thompson, 1995, p.82). On ne saurait mieux décrire le rôle du producteur de contenus à l'intention d'un public de masse. Or, Thompson démontre peu d'intérêt pour ces acteurs qui sont pourtant engagés, avec les membres du public, dans ce qu'il qualifie de relation de dépendance réciproque marquée par une profonde incertitude (Thompson 1995, p.95). En effet, son analyse de la production de contenus est peu développée, l'auteur s'attardant uniquement aux dispositifs techniques et rhétoriques par lesquels les acteurs qui en assurent la performance affichent leur souci (awareness) pour l'auditoire et leur font éventuellement une place dans celle-ci. ${ }^{3}$ En somme, Thompson discute de la quasi-interaction médiée uniquement en ce qu'elle implique un auditoire empiriquement vérifiable (en direct, en studio). Son modèle ne fait pas de place aux situations dans lesquelles le public ne constitue pas tout-à-fait - ou pas encore - un groupe social dont l'existence peut se vérifier empiriquement.

Si les médias de masse sont une caractéristique propre à la modernité dont l'avènement suscite un réaménagement des relations sociales et permet, avec la quasi-interaction médiée, la coordination d'individus dispersés dans le temps et l'espace, il y a lieu d'en saisir les manifestations non seulement au niveau de la réception (c'est-à-dire les contextes dans lesquels les individus sont interpelés comme récepteurs ou usagers), mais aussi en production. Les modalités de ce réaménagement et de cette coordination sont explorées dans ce qui suit.

\subsection{Réflexivité, familiarité et connaissances tacites}

Certains théoriciens des médias font de l'anticipation du public la clé de la coordination production-réception (McQuail, 1987; Gitlin, 1983; Dornfeld, 1998) sans pour autant idéaliser l'interaction directe producteurs-publics. Ils permettent de tempérer l'idée que les producteurs ne côtoient leurs publics que

\footnotetext{
3 Thompson présente l'exemple de l'émission de la BBC Question Time. Son analyse fait donc écho à Livingstone and Lunt (1994) et, plus largement, aux approches qui se penchent sur l'implication directe de publics en chair et en os.
} 
Philippe ROSS

dans des situations contrôlées en reconnaissant que la production et la réception se chevauchent de façons plus ou moins prévisibles. Ce chevauchement se manifeste dans les intuitions et connaissances tacites des producteurs (Ross, 2011b; Zafirau, 2009), dans leur recours au « gros bon sens »(Caldwell, 2008; Dornfeld, 1998; Gitlin, 1983), et dans leurs croyances (Ross, 2011b) au sujet du public, qui interviennent régulièrement en production.

Par exemple, les producteurs de télévision publique observés par Dornfeld (1998) font des suppositions quant à l'identité de leurs publics et celles-ci informent tant la conception d'émissions nouvelles que les décisions plus techniques concernant, par exemple, le cadrage et le montage. Gitlin (1983) a quant à lui montré que les producteurs de télévision dans les grands studios hollywoodiens interprètent les données statistiques sur leurs publics selon leurs goûts personnels et leurs interactions avec des amis et parents jugés représentatifs du public anticipé. Autrement dit, les études de marché sont utiles mais elles ne parlent jamais d'elles-mêmes; elles ne font sens qu'à l'aune de l'expérience. Dans la même veine, Zafirau (2009) et Auteur (année) ont démontré que les producteurs de contenus jeunesse invoquent leur expérience avec leurs propres enfants pour justifier certaines décisions prises en production. Bien que le recours à de telles connaissances du public n'aient rien de scientifique, elles ont le statut d' « atout » ou de « frime » (trump card) (Ross, 2011b, p.9; Caldwell, 2008, p.336), en ce qu'on leur confère une crédibilité et une autorité telles qu'elles peuvent supplanter tout autre argument au sujet du public, y compris ceux de spécialistes reconnus.

En sociologie des sciences et techniques, les mécanismes réflexifs par lesquels les producteurs recourent à leur expérience sociale ordinaire font partie des «techniques implicites» par lesquelles l'usager est engagé dans la production de technologies (Akrich, 1995). Certains décrivent ces techniques comme contraignantes et nuisibles aux visées généralistes des producteurs (Akrich, 1995; Oudshoorn et al., 2004; Williams et al., 2005). D'autres cependant les voient comme moyen privilégié de coordonner les acteurs (Bardini, 2000; Hennion, 1989), particulièrement aux stades précoces de la conception (Ross, 2011a; Nicoll, 2000), où l'on cherche d'abord et avant tout à établir la viabilité d'un projet, c'est-à-dire la probabilité que celui-ci résonne auprès d'un public, quel qu'il soit.

Quoi qu'il en soit de leurs impacts positifs ou négatifs, les mécanismes réflexifs attirent l'attention vers les situations sociales dans lesquelles producteurs et récepteurs éventuels interagissent et ce, indépendamment de l'entreprise de production dans laquelle elles sont subséquemment évoquées. En effet, ils sont les indicateurs d'une relation producteur-public existant sinon complètement « à l'état sauvage », du moins bien en amont des situations 
Coordination entre production et réception :

de l'interaction directe à la quasi-interaction médiée

contrôlées et du cadre instrumental prônés par les études critiquées plus haut. Cette relation peut être comprise à l'aune de la typologie de la formation des auditoires de McQuail, qui permet une conception plus ouverte des possibilités d'interaction entre producteurs et publics susceptibles d'avoir un impact sur la production.

\subsection{La relation circulaire entre production et réception}

McQuail (1987) a proposé une réflexion sociologique sur la relation entre émetteurs et récepteurs à partir de modèles canoniques de la communication interpersonnelle, qu'il a transposés à la production en milieu institutionnel afin de comprendre les pratiques des acteurs qui s'y trouvent et la manière dont ils appréhendent leurs publics. II accorde une attention particulière à l'ontologie de l'auditoire et ce qu'il appelle son "double caractère " : "audiences are both a cause of, and a response to, a supply of messages " (McQuail, 1987: 215). Ceci fait écho à la logique circulaire mise en lumière par Gitlin (1983) :

If we think of popular taste as a liquid brew of conventional expectations, themes and desires, held in suspension, then we may think of actual television programs as solid precipitates that suppliers and executives adapt and shape. (...) Once these precipitates form, a mass audience recognizes some of them as conforming to the contours of their desires. In that sense, only after the fact, the executives are half entitled to say that they are 'giving people what they want.' Another large mass of viewers might have wanted something else, but this particular mass is satisfied to want, or at least tolerate, what they are getting, from among the possibilities that slipped out of suspension (Gitlin, 1983, pp.29-30).

C'est précisément cette dynamique qui évoque la quasi-interaction médiée de Thompson, dans la mesure où elle traduit une relation entre groupes dispersés dans le temps et l'espace qui n'est pas dialogique, étant opérée par l'un seul de ces groupes, en l'occurrence les producteurs. En effet, ceux-ci ont beau prétendre n'être que des «instruments de la volonté populaire " (Gitlin, 1983, p.24), leurs produits ne sont pas pour autant le résultat direct des besoins du public. II faudrait plutôt les concevoir comme des "précipités ", c'est-à-dire le résultat matériel de débats parmi les producteurs autour duquel les dispositions de certains publics peuvent ensuite se cristalliser.

Pour McQuail (1987), le "double caractère » de l'auditoire sert de base à une typologie de la formation des auditoires qui distingue deux grands groupes selon leur origine : "les médias comme source" ('media as source') et "la 


\section{Philippe ROSS}

société comme source» ('society as source'). ${ }^{4}$ Ceux-ci correspondent respectivement à l'auditoire en tant que collectivité formée en réponse aux canaux ou contenus médiatiques, et à partir de forces sociales existant par ailleurs (McQuail, 1987, p.215). Le premier type (media origination) suppose que la relation entre émetteur et récepteur est formée par le biais du médium, entre producteurs et consommateurs d'un produit existant ou en développement. Le second type (society origination) suppose au contraire qu'une relation se forme dans des contextes sociaux qui existent en amont d'un artéfact particulier, menant éventuellement à la création d'un nouvel artéfact ou l'appropriation d'un artéfact existant jugé approprié et, par le fait même, à l'instauration d'une relation entre récepteurs et producteurs.

La notion de public aux origines sociales (society-originated audience) admet donc la possibilité d'une relation producteur-public qui ne dépende ni de l'existence d'un artéfact particulier, ni de celle de récepteurs réels ou effectifs comme c'est le cas des approches critiquées dans la première partie de cet article. Cependant, même si McQuail fait grand état du double caractère de l'auditoire et de la nature circulaire de son rapport aux producteurs, sa typologie maintient une distinction plutôt rigide entre origines sociales et origines médiatiques de l'auditoire. Elle cadre donc mal avec ce que les travaux en production studies et en sociologie des sciences et techniques nous apprennent sur l'usage de techniques implicites et explicites pour engager les publics. Ces mécanismes réflexifs démontrent qu'en pratique, il s'opère un croisement entre l'auditoire aux origines sociales et l'auditoire aux origines médiatiques. En effet, dans une situation d'incertitude quant aux récepteurs/usagers éventuels de leurs artéfacts et devant le besoin d'interpréter des études de marché qui ne parlent pas d'elles-mêmes - manifestations d'un auditoire aux origines médiatiques -, les producteurs font confiance à des individus issus de leurs relations interpersonnelles pour agir comme représentants du public, ou usagers par procuration. Ces derniers constituent selon nous un auditoire aux origines

\footnotetext{
4 La typologie comporte quatre catégories : «I. Groupe ou public (Group or Public): Concomitant avec un groupe social existant (e.g. communauté, minorité politique, religieuse ou ethnique) et partageant des caractéristiques telles le lieu géograhique, la classe sociale, la politique, la culture, etc. II. Regroupement de gratification (Gratification set): Formé sur la base d'un objectif ou besoin individuel existant indépendamment du médium; en lien, par exemple, avec un sujet politique ou social, un besoin en matière d'information ou pour une gratification émotionnelle. III. Groupe ou sous-culture d'amateurs (Fan Group or Taste Culture): Formé sur la base d'un intérêt pour un type particulier de contenu (ou genre) ou d'une attirance envers une personnalité publique particulière ou d'une préférence culturelle/intellectuelle particulière. IV. Auditoire d'un médium (Audience of a medium): Recruté, et maintenu par habitude ou loyauté, par une source médiatique particulière e.g. journal, magazine, réseau de radio ou de télévision. » (McQuail, 1987, p.222; traduction libre). Les deux premiers types relèvent de "society as source »; les deux derniers de «media as source».
} 
Coordination entre production et réception :

de l'interaction directe à la quasi-interaction médiée

sociales ${ }^{5}$, qui joue toutefois un rôle occulté par McQuail : il permet de combler la "distance sociale " (Thompson, 1995; Zafirau, 2009) qui sépare un groupe employé à la production d'artéfacts pour un autre qui ne peut être observé ou engagé directement. 6

Le rôle du producteur dans une telle dynamique s'apparente à celui du praticien réflexif qui déploie la "réflexion-en-action» (reflection-in-action) (Schön, 1983) pour combler le fossé qui se crée, en pratique, entre connaissances théoriques générales et contexte particulier nouveau et incertain. ${ }^{7}$ Schön soutient que la réflexion-en-action est déclenchée par l'intuition et les connaissances tacites du praticien, et que celles-ci constituent un processus par lequel il modèle «l'inconnu sur le familier » (modelling the unfamiliar on the familiar, Schön 1983: 184). Une telle conception cadre bien avec les moyens par lesquels les producteurs d'artéfacts et de contenus mobilisent leur connaissance du public anticipé. Elle reflète plus particulièrement l'usage des techniques implicites qui permet aux producteurs de simuler la présence du public en évoquant des individus et groupes qui leurs sont familiers. La pertinence de ce public putatif peut subséquemment s'avérer ou s'infirmer, au moment d'essais et de focus groups, par exemple. Mais qu'à cela ne tienne; sa seule évocation sert de base à l'action et rend possible les décisions en l'absence du public effectif et dans des contextes où l'incertitude pourrait autrement mener soit à réifier le public, soit à la paralysie.

En définitive, si la quasi-interaction médiée est bien un type d'interaction propre à la communication de masse, elle décrit la relation producteurs-publics de masse telle qu'elle est opérée par les producteurs, et pas seulement telle qu'elle est vécue par les récepteurs ou usagers. Loin de se fier uniquement à

\footnotetext{
5 L'un des évaluateurs anonymes de la version orignale de ce texte s'interroge sur une telle lecture, affirmant que l'individu a qui fait confiance le producteur peut tout autant appartenir à un auditoire aux origines sociales qu'à un public constitué autour d'un média. II s'agit d'une remarque pertinente. Mais selon nous, il importe peu que cet individu puisse appartenir à un public constitué autour d'un média $(x)$, dans la mesure où sa relation avec le producteur existe dans un contexte social indépendent du produit en développement $(y)$, justifiant ainsi l'origine sociale de l'auditoire. Dans tous les cas, la distinction entre les deux types d'origines n'est pas aussi étanche que ne le suggère la typologie de McQuail.

6 Ces mécanismes implicites permettent ainsi de concevoir l'influence indirecte de la réception et des récepteurs sur la production, à la manière du modèle à deux-étages de Katz et Lazarsfeld (1957), mais inversé (Ross, 2012a): les relations interpersonnelles des producteurs agissent comme filtre ou tampon entre le contexte de production et celui de la réception qu'ils cherchent à appréhender.

7 Schön développe sa conception du praticien réflexif en réaction à « l'épistémologie positiviste de la pratique " (Schön, 1983, p.31) qui, depuis le $18^{\mathrm{e}}$ siècle, conduit à penser la relation entre la connaissance scientifique et sa mobilisation comme « the instrumental adjustment of means to ends » (Schön, 1983, p.23).
} 
Philippe ROSS

l'interaction directe avec les récepteurs/usagers effectifs de leurs créations dans des situations contrôlées, les producteurs coordonnent production et réception en conjuguant des groupes dispersés dans le temps et l'espace: la masse putative à laquelle les confronte la situation de production, et les relations interpersonnelles qu'ils invoquent pour s'en rapprocher.

\section{Conclusions}

Tant les praticiens que les chercheurs dans le domaine des médias s'empressent, depuis un certain temps, à étudier des contextes de réception et d'usage qui sont de plus en plus caractérisés par des comportements 'de production'. En font foi non seulement les études portant sur les nouvelles formes de collaboration rendues possibles avec le Web 2.0, mais aussi celles qui mettent l'accent sur le rôle actif des usagers dans la mise en forme technique. Mais, pour reprendre la question posée en introduction : la nature de la production en tant qu'activité sociale orientée vers le public doit-elle conduire à favoriser le rôle actif de ce dernier en production?

Bien qu'elles soient utiles à la compréhension du rapport entre production et réception, ces approches se développent en l'absence d'une conception adéquate, selon nous, des aspects sociaux de la production et, plus particulièrement, de la façon dont celle-ci s'inscrit en continuité - plutôt qu'en rupture - avec les relations sociales des individus qui y oeuvrent.

Pour se convaincre de l'ampleur du problème, il suffit de considérer l'affirmation, colportée dans les discours populaire et scientifique, à l'effet que les «gens ordinaires » sont aujourd'hui en mesure de diffuser des contenus à un public de masse et ainsi d'exercer une forme de «pouvoir symbolique » (Thompson, 1995) - un rôle vu traditionnellement comme l'apanage des institutions médiatiques. Une telle opposition entre gens ordinaires et institutions médiatiques laisse sous-entendre que les individus désormais en mesure d'agir comme communicateurs de masse ne possèdent aucune compétence particulière; et/ou que les professionnels de la production ne sont pas, eux, ordinaires - ils seraient extraordinaires. Or, la discussion menée dans les pages qui précèdent montre bien que ce type de distinction binaire ne saurait tenir la route, précisément parce qu'il conçoit à tort d'une rupture entre les espaces sociaux de la production et de la réception et qu'il mobilise une conception trop instrumentale de leur médiation par les acteurs de la production. À l'inverse, nous avons démontré que producteurs et récepteurs éventuels fréquentent en fait des espaces sociaux qui se chevauchent ; et que la médiation entre les deux tient à des mécanismes informels, voire implicites. Si bien que, lorsqu'il s'agit de comprendre ou d'anticiper le public - ce que d'aucuns reconnaissent comme la compétence-clé en production - les soi-disant gens ordinaires, membres du 
Coordination entre production et réception :

de l'interaction directe à la quasi-interaction médiée

public de masse, peuvent être perçus comme ayant des connaissances que les producteurs cherchent par tous les moyens à acquérir : quant aux besoins, goûts, préférences et dispositions qui sont les conditions au succès de toute production.

Ceci évoque le rôle des intermédiaires dans les phénomènes collectifs comme les paniques médiatiques (Livingstone, 1996), la diffusion des innovations (Valente, 1995) et, dans le cas qui nous concerne, la stabilisation de réseaux sociotechniques. Dans de telles situations, où les acteurs sont confrontés au risque, à l'incertitude et à l'incapacité d'observer les résultats de l'action individuelle à une échelle macro, ils ne se tournent pas vers d'autres individus - soient-ils leaders d'opinions, usagers effectifs ou récepteurs présumés - pour obtenir d'eux des certitudes quant à la meilleure action à prendre. Plutôt, ils se tournent vers des intermédiaires dont ils présument qu'ils savent mieux qu'eux quelle action est susceptible d'engendrer le résultat escompté. Le contexte de production de fait pas exception à cette règle. Le défi pour les producteurs n'est pas tant d'acquérir une connaissance parfaite des goûts, préférences et dispositions du public anticipé; il est d'acquérir plus de connaissance, et de sources perçues comme fiables. Dans un tel contexte, la connaissance est relative, pas absolue. Cette distinction fort importante se perd avec la tendance qui consiste à faire de l'interaction directe en face-à-face le paradigme de la relation entre producteurs et leurs publics. Comme si le public de masse et ses besoins étaient une réalité empirique saisissable - pour peu que les producteurs utilisent les bons outils, les bonnes méthodes et les bons échantillons d'usagers afin de simuler, dans une situation de laboratoire contrôlée, le public anticipé.

La réflexion théorique développée dans cet article a cherché à défendre l'idée que la relation entre média et public de masse, telle qu'elle se manifeste en production, n'est pas plus directe qu'elle ne l'est en réception. Comme dans les contextes de réception, elle est médiée par l'expérience et les relations sociales des individus - en l'occurrence les producteurs. Dans le contexte médiatique contemporain, une telle perspective peut sembler contre-intuitive. Or, il y a - et il y aura toujours - besoin de jeter un éclairage sur ce qui se passe en l'absence des usagers ou récepteurs effectifs, même si ceux-ci sont de plus en plus actifs et impliqués en production. Les formes émergentes de participation telles le réseautage social, le contenu généré par les usagers, le crowdsourcing et le crowdfunding montrent bien qu'il y a une demande très forte pour l'information produite par les membres du public « ordinaires » et à laquelle les producteurs professionnels, seuls, ne peuvent répondre. 
Philippe ROSS

Mais cette logique explique également le développement des moteurs de recherche et de recommandation qui exploitent les contributions plus tacites du public par le biais du forage de données. Dans tous ces cas, la participation du public est médiée par les outils, plateformes et algorithmes - autant de technologies qui incarnent des visions particulières du public qui ne sont ni transparentes, ni inévitables. Cela nous rappelle la nécessité de scruter le travail des producteurs au sens traditionnel : ces individus qui conçoivent les artéfacts et les contenus qui se fondent à notre vie quotidienne et dont le travail se résume au commerce des publics, soient-ils effectifs ou présumés.

\section{Références Bibliographiques}

AKRICH, M., 1995, « User Representations: Practices, Methods and Sociology », dans RIP, A., T. MISA, et J. SCHOT (dir.) Managing Technology in Society: The Approach of Constructive Technology Assessment, New York: St. Martin's Press, pp. 167-184.

AKRICH, M., 1998, «Les utilisateurs, acteurs de l'innovation », Éducation permanente, No 134, pp. 79-89.

AKRICH, M., 1992, "The de-scription of technical objects ", dans Shaping technology/building society: Studies in sociotechnical change, BIJKER, W.E. et J. LAW (dir), Cambridge, MA: The MIT Press, pp. 205-224.

BARDINI, T., 2000, Bootstrapping: Douglas Engelbart, coevolution, and the origins of personal computing. Stanford, CA: Stanford University Press.

BEIRNE, M., H. RAMSAY et A. PANTELI, 1998, « Participating Informally: Opportunities and Dilemmas in User-Driven Design », Behaviour \& Information Technology, Vol. 17, No. 5, pp. 301-310.

BELL, A., 2012, "'ScienceBlogs is a high school clique, Nature Network is a private club': Imagining the communities of online science ", Canadian Journal of Media Studies, numéro thématique (automne), pp. 240-265.

BENNETT, W. L. et J. B. MANHEIM, 2006, « The One-Step Flow of Communication ", Annals of the AAPSS, 608, pp. 213-232.

BOYD, d., 2007, "Why youth <heart> social network sites. The role of networked publics in teenage social life ", dans Youth Identity and Digital Media, D. Buckingham (dir), Cambridge, MA: The MIT Press, pp. 119-142. 
Coordination entre production et réception :

de l'interaction directe à la quasi-interaction médiée

BRAKE, D., 2009, « As if nobody's reading'?: The imagined audience and sociotechnical biases in personal blogging practice in the UK ", thèse de doctorat inédite, London School of Economics and Political Science.

BROWN, J.S. et P. DUGUID, 1994, « Borderline Issues: Social and Material Aspects of Design ", Human-Computer Interaction, Vol. 9, pp. 3-36.

CALDWELL, J.T., 2008, Production culture: Industrial reflexivity and critical practice in film and television. Durham: Duke University Press.

CALLON, M., 1986, " Some elements of a sociology of translation:

Domestication of the scallops and the fishermen of Saint Brieuc Bay », dans LAW, J. (dir), Power, Action and Belief: A New Sociology of Knowledge? Sociological Review Monograph 32. London: Routledge and Kegan Paul, pp. 196-233.

CASTELLS, M., 2009, Communication Power. Oxford: Oxford University Press.

COULDRY, N., 2008, " Mediatization or mediation? Alternative understandings of the mediated space of digital storytelling », New Media \& Society 10(13): pp.373-391.

DEUZE, M., 2007, Media work. Cambridge, UK: Polity.

DORNFELD, B., 1998, Producing public television, producing public culture. Princeton, NJ: Princeton University Press.

EDQUIST, C., 1997, « Systems of Innovation Approaches - Their Emergence and Characteristics ", dans Systems of Innovation: Technologies, Institutions and Organizations, EDQUIST, C. (dir), London and Washington: Pinter, pp. 1-35.

FLICHY, P., 1995, L'innovation technique. Récents développements en sciences sociales. Vers une nouvelles théorie de l'innovation, Paris: Éditions La Découverte.

FULK, J. et G. DESANCTIS, 1995, «Electronic Communication and Changing Organizational Forms », Organization Science, Vol. 6, No. 4, pp. 337-349.

GITLIN, T., 1983, Inside Prime Time, New York: Pantheon Books.

HENNION, A., 1989, " An intermediary between production and consumption: the producer of popular music », Science, Technology and Human Values 14: pp. 400-424. 
Philippe ROSS

HERMIDA, A. et N. THURMAN, 2008, " A Clash of Cultures: The integration of user-generated content within professional journalistic frameworks at British newspaper websites ", Journalism Practice 2(3), pp. 343-356.

HESMONDHALGH, D. et S. BAKER, 2011, Creative labour. Media work in three cultural industries. London: Routledge.

HINE, C., 2001, «Web Pages, Authors and Audiences: The Meaning of a Mouse Click », Information, Communication \& Society, Vol. 4, No. 2, pp. 182198.

JENKINS, H. 2006, Fans, bloggers, and gamers: exploring participatory culture. New York: New York University Press.

JOUËT, J., 2003, « La Pêche aux internautes », Hermès 37, pp. 203-211.

KATZ, E., 1957, « The two-step flow of communication: An up-to-date report on a hypothesis », Public Opinion Quarterly 21: pp.61-78.

KAUFMANN, L., 2008, «La société de déférence: médias, médiations et communication », Réseaux no. 148/149, pp. 79-116.

LATOUR, B., 1989, La science en action. Introduction à la sociologie des sciences, Paris: Gallimard.

LIEVROUW, L. A., 2002, « Determination and Contingency in New Media Development: Diffusion of Innovations and Social Shaping of Technology Perspectives ", dans Handbook of New Media: Social Shaping and Consequences of ICTS, LIEVROUW, L. A. et S. LIVINGSTONE (dir), London, Thousand Oaks, CA and New Delhi: Sage, pp. 183-199.

LIVINGSTONE, S., 2004, "The Challenge of Changing Audiences: Or, What is the Audience Researcher to do in the Age of the Internet? ", European Journal of Communication, Vol. 19, No. 1, pp. 75-86.

LIVINGSTONE, S., 1996, "On the Continuing Problem of Media Effects », dans Mass Media and Society, CURRAN, M. et J. GUREVITCH (dir), London and New York : Arnold, pp. 305-324.

LIVINGSTONE, S. et P. K. LUNT, 1994, Talk on Television: Audience Participation and Public Debate, London and New York: Routledge.

LUNDVALL, B.-A., 1992, National Systems of Innovation: Toward a Theory of Innovation and Interactive Learning, London and New York: Pinter Publishers. 
Coordination entre production et réception :

de l'interaction directe à la quasi-interaction médiée

MACKAY, H., C. CARNE, P. BEYNON-DAVIES et D. TUDHOPE, 2000,

"Reconfiguring the User: Using Rapid Application Development », Social

Studies of Science, Vol. 30, No. 5, pp. 737-757.

MARCUS, G.E., 1998, Ethnography through thick and thin. Princeton, NJ: Princeton University Press.

MARWICK, A.E. et d. BOYD, 2011, «I tweet honestly, I tweet passionately: Twitter users, context collapse and the imagined audience ", New Media \& Society 13(1): pp. 114-133.

MAYER, V., 2011, Below the line: producers and production studies in the new television economy. Durham: Duke University Press.

MAYER, V., M. J. BANKS et J. T. CALDWELL (dir)., 2009, Production Studies: Cultural Studies of Media Industries. London: Routledge.

MCQUAIL, D., 1987, Mass communication theory: An introduction (2e édition). London: Sage.

MONGE, P. R., J. FULK, M. E. KALMAN, A. J. FLANAGIN, C. PARNASSA et S. RUMSEY, 1998, "Production of Collective Action in Alliance-Based Interorganizational Communication and Information Systems », Organization Science, Vol. 9 No. 3, pp. 411-433.

NICOLL, D.W., 2000, " Users as currency: Technology and marketing trials as naturalistic environments », The Information Society 16: pp. 303-310.

OUDSHOORN, N., E. ROMMES and M. STIENSTRA, 2004, "Configuring the User as Everybody: Gender and Design Cultures in Information and Communication Technologies ", Science, Technology \& Human Values 29(1): pp.30-63.

PARISER, E., 2011, The Filter Bubble. What the Internet is Hiding From You. New York: The Penguin Press.

PINCH, T. et N. OUDSHOORN (dir)., 2003, How Users Matter: The CoConstruction of Users and Technology. Cambridge, MA: The MIT Press.

PINCH, T. J. et W. E. BIJKER, 1984, « The social construction of facts and artefacts: or how the sociology of science and the sociology of technology can benefit each other ", Social Studies of Science, Vol. 14, pp. 399-441.

ROSS, P., 2012a, « Were Producers and Audiences Ever Separate? Conceptualizing Production as Social Situation "Television and New Media, OnlineFirst Version of Record: 24 August 2012. 
ROSS, P., 2012b, « What Is Distinctive About Mediation? Experience and Expertise in Media ", Sociology Compass 6(10), pp. 781-792.

ROSS, P., 2011a, « Problematizing the User in User-Centred Production: A New Media Lab Meets its Audiences ", Social Studies of Science 41(2): pp. 251-270.

ROSS, P., 2011b, «Is There an Expertise of Production? The Case of New Media Producers », New Media \& Society 13(6): pp.912-928.

SCHÖN, D., 1983, The Reflective Practitioner: How Professionals Think in Action, Aldershot: Ashgate.

SCHUMPETER, J. A., 1934, The Theory of Economic Development, Cambridge, MA: Harvard University Press.

SILVERSTONE, R., 2005, "The sociology of mediation and communication », dans The Sage Handbook of Sociology, CALHOUN, C., C. ROJEK and B. TURNER (dirs), London: Sage, pp. 188-207.

STAR, S. L. et G. BOWKER, 2002, " How to Infrastructure ", dans Handbook of New Media: Social Shaping and Consequences of ICTs, L. A. LIEVROUW et S. LIVINGSTONE (dir), London, Thousand Oaks and New Delhi: Sage Publications, pp. 151-162.

STEWART, J. et R. WILLIAMS, 2005, "The wrong trousers? Beyond the design fallacy: Social learning and the user ", dans ROHRACHER, H. (dir) User Involvement in Innovation Processes. Strategies and Limitations from a Socio-technical Perspective. Munich: Profil-Verlag.

TAPSCOTT, D. et A.D. WILLIAMS, 2010, Macro-Wikinomics: Rebooting Business and the World. New York: Portfolio.

THOMPSON, J. B., 1995, The Media and Modernity: A Social Theory of the Media, Cambridge: Polity Press.

TUROW, J., 2012, The Daily You. How the Advertising Industry is Defining Your Identity and Your World. New Haven and London: Yale University Press.

VALENTE, T. W., 1995, Network Models of the Diffusion of Innovations, Cresskill, NJ: Hampton Press Inc.

VON HIPPEL, E., 1986, "Lead Users: A Source of Novel Product Concepts ", Management Science, Vol. 32, No. 7, pp. 791-805.

WILLIAMS, R., J. STEWART et R. SLACK, 2005, Social Learning in Technological Innovation: Experimenting with Information and Communication Technologies. Cheltenham: Edward Elgar. 
Coordination entre production et réception :

de l'interaction directe à la quasi-interaction médiée

ZAFIRAU, S., 2009, " Audience knowledge and the everyday lives of cultural producers in Hollywood ", dans Production studies: Cultural studies of media industries, V. MAYER, M. J. BANKS et J.T. CALDWELL (dir), London:

Routledge, pp. 190-202. 